\title{
Towards Improving Trust in Context-Aware Systems by Displaying System Confidence
}

\author{
Stavros Antifakos \\ Dep. of Computer Science \\ ETH Zurich, Switzerland \\ antifakos@inf.ethz.ch
}

\author{
Nicky Kern, Bernt Schiele \\ Dep. of Computer Science \\ Darmstadt University of \\ Technology, Germany \\ \{nicky.kern,schiele\}@ \\ informatik.tu- \\ darmstadt.de
}

\author{
Adrian Schwaninger \\ Max Planck Institute for \\ Biological Cybernetics \\ 72076 Tübingen, Germany \\ adrian.schwaninger@ \\ tuebingen.mpg.de
}

\begin{abstract}
For automatic or context-aware systems a major issue is user trust, which is to a large extent determined by system reliability. For systems based on sensor input which are inherently uncertain or even uncomplete there is little hope that they will ever be perfectly reliable. In this paper we test the hypothesis if explicitly displaying the current confidence of the system increases the usability of such systems. For the example of a context-aware mobile phone, the experiments show that displaying confidence information increases the user's trust in the system.
\end{abstract}

\section{Categories and Subject Descriptors}

H.5.2. [Information Interfaces and Presentation]: User Interfaces; H.1.2. [Models and Principles]: User/Machine Systems-Human factors

\section{Keywords}

Uncertainty, Context-Aware Systems, Trust, System Confidence

\section{General Terms}

Human Factors

\section{INTRODUCTION}

When interacting with automatic systems such as intelligent agents or context-aware systems, the user's trust in those systems is a major factor. It depends to a large extent on the reliability of the system. As many context-aware systems in the domain of ubiquitous and wearable computing are based on sensor input, there is little hope that they will ever be perfectly reliable. Thus these systems must find a way to handle the inherent uncertainty of their sensory input and integrate it into the interaction with the user.

Permission to make digital or hard copies of all or part of this work for personal or classroom use is granted without fee provided that copies are not made or distributed for profit or commercial advantage and that copies bear this notice and the full citation on the first page. To copy otherwise, to republish, to post on servers or to redistribute to lists, requires prior specific permission and/or a fee.

MobileHCI'05, September 19-22, 2005, Salzburg, Austria

Copyright 2005 ACM 1-59593-089-2/05/0009 ...\$5.00.
The ability to extend interaction with the user beyond the actual computer and its user is often considered key ingredient for ubiquitous and wearable computing. Such context information allows the computer to seamlessly adapt its behaviour to the situation of the user and his environment. Including context information is a non-trivial task. While external things, such as objects, the environment, and people, might be relatively simple to capture internal things such as people's current interests, objectives, and the state of the activity people are pursuing, are extremely difficult to capture (see Greenberg [10]). For some information, e.g. human aspects, it might not be possible at all to capture it through technological means (Bellotti and Edwards [2]).

How people interact with context aware systems that are not perfectly reliable has only been analyzed sparsely [21]. However, many analogies between context-aware systems and automated systems used in various industries can be found. For example systems have been built to autonomously fly planes from take-off to landing or control entire power plants. Typically a supervisor controls the automated system and decides when to use it or not. Muir [17] is one of the first researchers to look at this decision process between supervisors and automated systems. She verifies the hypothesis proposed by Sheridan et al. [25] that the supervisor's intervention behavior is based upon his/her trust in automation. Context-aware systems similarly follow the goal of automating tasks or adapting to certain situations in everyday life. Whether a person decides to use such an automated system or a context-aware system is a similar decision process. Similar parallels can also be drawn with the field of intelligent software agents. Interestingly, Maes [15] argues that the user's trust is one of the two major issues besides agent performance - in agent design.

In this paper we propose and explore a particular way of user feedback in order to increase the user's trust in a context-aware system. The proposal is based on the fact that users are used to and highly successful in dealing with unreliable and uncertain information throughout their daily lives. We propose to display system reliability explicitly and leverage from the user's ability to choose the appropriate action.

Trust is, being highly subjective, inherently hard to measure. As it is an internal 'state' of the user, there is no way of measuring it directly. Furthermore, trust is built up over time and changes with the use of a system. There is a continuum of initial trust the user has before having any 
expererience with a system, short-term trust that builds up over the first interactions with a system and long-term trust that develops with the continuous use of a system over a longer period of time. In this paper we investigate shortterm trust. As trust is easily lost, but gained only slowly (see Tiernan et al. [26]) this is a crucial phase of acquiring long-term trust in a system. Furthermore, this level of trust is comparatively easy to measure after having some initial experience with a system.

The example of a context-aware mobile phone is used in this paper, as it is easily imaginable by study participants. The vision is that a context-aware mobile phone chooses the correct notification modality depending on the current situation. The user can decide either to trust the system or simply to override the automation before entering a critical situation, in a similar manner as we would today, entering a theater for example.

It is worth pointing out that recent research has shown the feasibility of such a context-aware mobile phone. As a matter of fact quite general notification systems have been designed and implemented that estimate the human's interruptability using a variety of sensors. Examples of such systems can be found in $[8,14,13]$ amongst others.

The following section reviews methods for dealing with the inherent uncertainty in context-aware systems. To understand the effects of our approach we then discuss the notion of trust in automated systems as it is presented in the literature. The principal contribution of this paper is a study in which we show the effect of displaying system confidence on user trust. This study is based on the scenario of a context-aware mobile phone. We summarize the paper and discuss extensions to this work.

\section{DEALING WITH UNCERTAINTY}

Many of today's context aware systems do not deal with uncertainty of context information. However, they could be extended to do so. Obviously, systems exist which explicitly model and use uncertainty during inference and decision making. Maybe the most advanced systems like the Lumiere project [10], the Lookout project [11] or the Activity Compass [22] are based on techniques such as Bayesian modeling and inference, utility, and decision theory. There is interesting efforts in the field of neurophysics to build (baysian) models of the user's mental state (see for example [20]).

In the context of ubiquitous computing it has been suggested, however, that modeling uncertainties and advanced inference mechanisms might not be enough. Starting from the observation that there are human aspects of context that cannot be sensed or inferred by technological means, Bellotti and Edwards [2] conclude that context systems cannot be designed simply to act on our behalf. Rather they propose that those systems will have to defer to users in an efficient and non-obtrusive way. They also present design principles which support intelligibility of system behavior and accountability of human users. Greenberg [10] also states that actions automatically taken by the system should be clearly linked to the respective context through feedback. Chalmers [4] even argues for "seamful rather than seamless design" to reveal the physical nature of the Ubicomp systems in, for example, the uncertainty in sensing and ambiguity in representations. Mankoff et al. [16] developed a toolkit that supports resolution of input ambiguity through mediation by building on various methods of error correction in user interfaces. More recently Newberger and Dey [18] have extended the Context Toolkit by a so-called enactor component that encapsulates application state and manipulation to allow users to monitor and control context-aware applications. Horvitz and Barry [12] extend their framework to also estimate the expected value of revealed information to enhance computer displays to monitor applications for a time-critical application at NASA. Williamson and MurraySmith [27] propose to display system uncertainty on the audio channel for closed-loop systems, but have not (yet) results on how this increases user performance in using the system.

All of the above-mentioned approaches offer solutions to deal with the inherent uncertainty problem of context information. What is common to all of them is to propose the use of different feedback mechanisms and to involve the user in various degrees and forms. While those approaches are well motivated in their respective application context, their effects on user's trust and system usability have not been evaluated.

\section{THE NOTION OF TRUST IN MACHINES}

Trust is a multifaceted concept that each person keeps in his mind. Trust is always with respect to a certain reference person. Even more, it is usually directed towards a single attribute of a person. When delegating a task to another person and we do not know and do not trust that person yet we are unlikely to give that person a highly critical task. Over time however, when we have built up some trust based on satisfying completion of several tasks we are much more willing to delegate more critical tasks to the respective person. Trust increases through positive experience and similarly decreases with negative experience.

The concept of having trust in machines can be observed in everyday life. One might be more or less comfortable using electronic systems such as navigation aids or cash-cards depending in the trust one has in those systems. Again, the level of trust often results from previous experiences, where the automatic system did or did not work as expected.

Muir [17] extended a model of trust between people, to trust between humans and machines. She argues that the notion of trust is necessary as humans can hardly ever have complete knowledge of the inner workings of automated systems. In order to make use of such systems, they have to rely on certain properties and they need to be able to expect certain behavior. Change of trust is a result of experience with a system. At the beginning of using an automated system, its predictability is an important factor which depends on three factors: firstly, the actual predictability of the automated machine's behavior; secondly, the human's ability to estimate the predictability of the machine's behavior; thirdly, the stability of the environment in which the system operates.

The human's ability to estimate the predictability of an automated system depends on how easy the system is to observe. Dzindolet et al. [6] show that study participants improve on their detection rates of soldiers in still images when explanations were given about the system's functioning, and when it might err. Similarly, Bubb-Lewis and Scerbo [3] argue that the only way of reducing uncertainty is by exchanging information between the automatic system and the human user. 
When automated systems are used over longer periods of time also dependability and faith are key factors in the formation of trust. Dependability describes trustworthiness across longer time periods. Faith describes the belief that the system will remain trustworthy in the future. How trust is affected by system errors has been evaluated in several research fields. In the field of automated notification systems Tiernan et al. [26] find that trust doesn't recover quickly after a wrong notification is made. Similar work was done in the domain of intelligent agents [7] and with a decision aid for a route planning task [5].

Clearly, the level of trust in the system influences the use and rejection of automated and context-aware systems. As Muir [17] argues and shows the usage of such a system will be optimal when the user's trust level adapts to the objective trustworthiness of the system. Here, the trustworthiness of the system is given by the system reliability. This process of adapting the level of trust is called calibration of trust.

Comparing this section about trust to the previous one about handling uncertainty, it is striking, how many methods of presenting feedback can be seen as an effort towards making systems more predictable. As the next sections show, our proposal to display system confidence is a simple, but effective, way of helping the human to predict the outcome of the system and to use the system more effectively.

\section{DISPLAYING SYSTEM CONFIDENCE}

We conducted a two-stage user study to investigate the effects of displaying system confidence in an automatic notification device. We collected videos of 47 prototypical situations (2 per situation). In a first experiment we assessed the criticality of the situations therein. Using this result we selected a smaller set of 30 situations based on criticality (see Section 4.2 below). We used those in the second experiment, which investigates the effects of availability of confidence cue, system confidence, and situation on the user's trust in the system.

The 47 prototypical situations (2 per situation) were selected to provide a diverse sample of everyday situations. Examples include studying in a library, buying gum at a kiosk, sitting in a tram, attending a lecture, or eating in various restaurants (McDonalds, students' restaurant, 'proper' restaurant). Two different 5 -second videos were recorded for each situation, resulting in a total of 94 videos.

The first experiment assesses people's modality preferences for each of the 94 videos. At the same time we ask the people to rate how critical it is that the preferred modality is actually used for notification. This experiment gives us the possibility to classify the situations in low, medium, and highly critical situations. We use this rating to select videos for the second experiment.

In the second experiment we show other participants a subset of the situations. For each situation we ask them to rate whether they would check the settings of an automatic system. After rating the situation a window pops up displaying the notification modality chosen by the automatic system. This modality is generated by taking the modality preferences from the first experiment and "changing" it slightly depending on the momentary simulated system confidence. In one experiment block the system confidence is displayed, in the other block no information about system confidence is given. In this way the effects of displaying

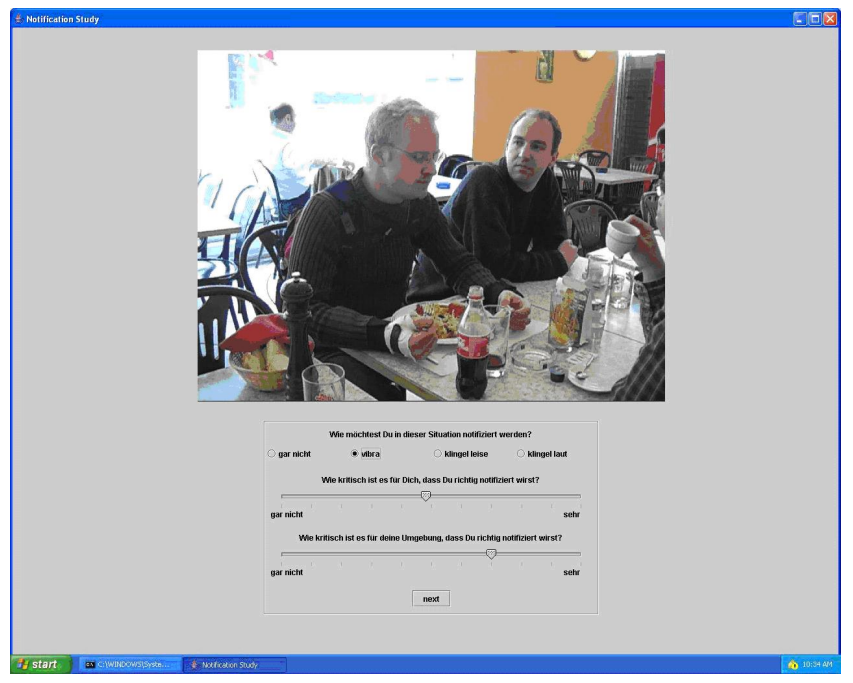

Figure 1: Screenshot of the first experiment: The first question allows the user to enter the preferred modality. The two slider controls allow the user to rate the criticality of the situation (for oneself and for the environment).

system confidence on the user's trust in the system can be evaluated.

\subsection{Experiment 1: Choice of Situations}

The goal of this experiment is to introduce a criticality ordering into the situations and to select a small set of situations for the second experiment. To determine the criticality, each participant's preferred modality is assessed. Criticality is defined by how critical it is to the participant that a context-aware system selects the preferred modality.

\subsubsection{Method and Procedure}

In this experiment each participant completed a series of trials to assess the preferred modality and the criticality for all situations. For each situation, shown as a 5 -second video, the participants were asked to answer the following question:

- With which modality would you like to be notified?

The following options were provided to answer this question: 'not at all', 'vibration mode', 'ring-tone', and 'ringtone loud'.

Following the model presented in [14] we assess the criticality of the situations separately for the user and the environment. The following two questions assess the criticality of a correct notification:

- How critical is it to you, that you are notified correctly in this situation?

- How critical is it to your environment, that you are notified correctly in this situation?

Both questions were rated on a slider control ranging from "not at all" to "very much". After a short introduction, a trial run with 4 situations was completed. After answering these questions the participants rated all 94 situation videos. 


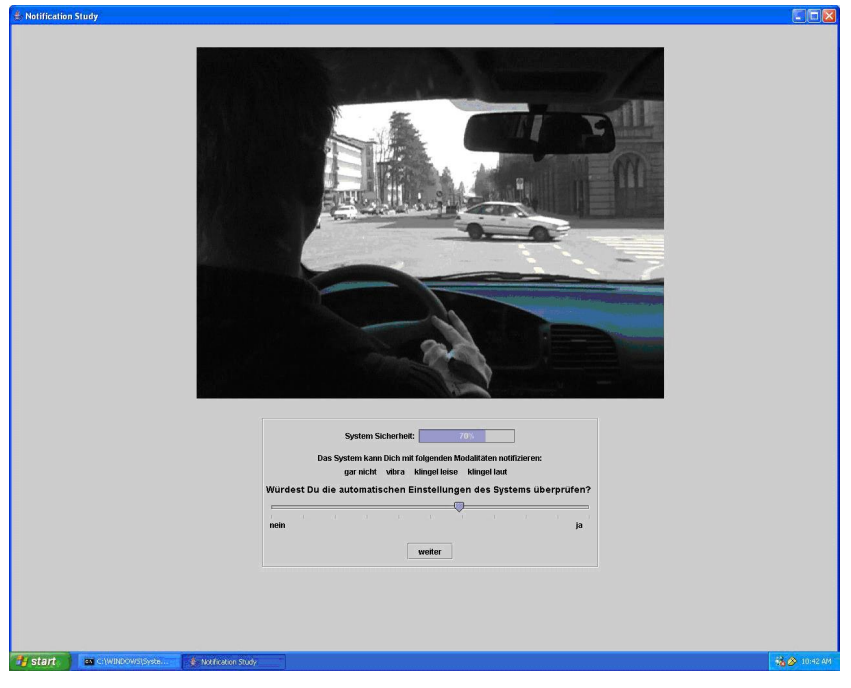

Figure 2: Screenshot of Experiment 2: System confidence is display as a graphical bar. The participants can rate whether they would check the modality selection of an automatic system on a continuous scale from no to yes. After pressing the 'next' button a message box pops up displaying the automatically chosen modality.

\subsubsection{Equipment and Participants}

The experiment was conducted using a personal computer running Windows XP with the screen resolution set at 1280x1024 on a TFT screen. A program was written to display the videos and to record the user's answers (see Figure 1). The Program covered the entire computer screen except the Windows task bar.

12 students from the Department of Psychology of the University of Zurich and the Computer Science Department of ETH Zurich participated in the first experiment. Half of the participants were female. Average age was 26 years with a standard deviation of 4.6 years. All participants reported to own a mobile phone, on average for 3.9 years. $50 \%$ of all participants use their phone several times per day.

\subsubsection{Results and Choice of Situations}

In the experiment both the personal and social criticality of each situation was measured by asking how critical a wrong modality is for oneself and for the people in the environment. In the work presented here we only make use of the personal criticality measure.

The personal criticality ratings for the 94 situations varied between .297 and .972 , which shows that the video clips covered the continuum of criticality quite well (a value of 0 corresponds to not at all critical, a value of 1 represents very critical).

\subsection{Experiment 2: User Trust}

The main goal of the second experiment is to evaluate the effects of displaying system confidence on the user's trust towards a context-aware mobile phone. Trust is measured by capturing how often the user would verify a modality the system has automatically derived. The assumption hereby is that when people trust the system to select the correct notification modality, there is no need to check the system's settings. In addition, one would assume that, the verification rate also depends on how critical a situation is.

We chose three sets of 10 situations based on the ratings of Experiment 1. The situations were sampled from the set of all videos such that means were equidistant and standard deviation was equal for the first two decimal places. The situations with low criticality (mean: 0.407, stddev: 0.068) included: sitting in a tram, standing in the elevator alone, looking at shop windows, buying a train-ticket at a machine etc.. The situations with medium criticality (mean: 0.633 stddev: 0.068) included: eating at MacDonald's, driving a car, buying a chewing gum at a kiosk, and others. Finally, the situations with a high criticality (mean: 0.869 stddev: 0.067) included: attending a lecture, studying at a university library, and working in a common computer lab amongst others.

\subsubsection{Design}

We used a within-participants design with three independent variables: situation, confidence-level, and cue-availability, i.e. whether or not system confidence information is displayed to the user.

The experiment was conducted in three blocks. In the first block the participant's preferred modalities are assessed using the setup from Experiment 1. Block 2 and 3 ask the question of how much the participant relies on a contextaware system. In one of these two blocks the confidence-cue is displayed whilst in the other block no information about system confidence is given. The independent variables situation and confidence-level are randomized in both blocks.

Block 2 and 3 make use of the previously chosen 30 different situations. These 30 video sequences were repeated at 3 different confidence levels. This resulted in 90 trials per block. Block order was counterbalanced across participants. Each situation was shown as a 5-second video. For each video the participants had to rate the following question, on a continuous scale from "no" to "yes":

- In this situation, would you check the modality automatically selected by the system?

After rating the question, and clicking on the "next" button, a pop-up message appears, displaying the notification modality that was automatically selected by the system. The notification modality is chosen in dependence of the randomly set system confidence-level. We varied the confidencelevel between 0.5, 0.7, and 0.9.

\subsubsection{Equipment and Participants}

The experiment was conducted using a personal computer running Windows XP with the screen resolution set to 1280x1024 on a TFT screen. A program was written to display the videos and to record the participant's answers (see Figure 2). The Program covered the entire computer screen except the Windows task bar.

14 students from the Department of Psychology of the University of Zurich and the Computer Science Department of ETH Zurich participated in the second experiment. Half of the participants were female. Average age was 26 years with a standard deviation of 3.6 years. All, except for one participant, reported to own a mobile phone, on average for 4.1 years. $57 \%$ of all participants indicated that they use their phone several times a day. None of these participants took part in the first experiment. 


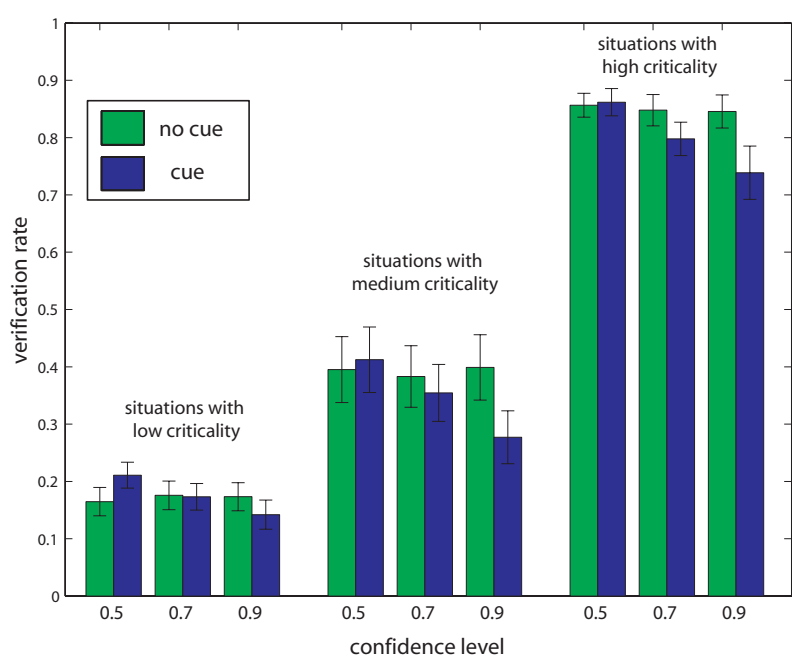

Figure 3: Verification rates with standard error for the three different types of situations and system confidence levels.

\subsubsection{Procedure}

First, participants are introduced into the notion of contextaware systems and the idea of a context-aware mobile phone. After that, the graphical user interfaces for the three experiment blocks are reviewed in detail. Prior to the experiment each participant completes an introductory run consisting of 4 situations for each block.

\subsubsection{Results}

Figure 3 shows the verification rates for the three categories of situations. As would be expected, it is clearly visible that the participants verify the context-aware system most often in highly critical situations. The figure further suggests that the verification rates are lower, in the high confidence case (0.9), when system confidence information is displayed. In contrast, the verification rates tend to be slightly higher in the low confidence (0.5) case.

The conventional cut-off of $p<.05$ was used for all tests of statistical significance. The verification rates were subjected to an analysis of variance (ANOVA) with the three following within-subject factors: cue-availability, confidence level $(0.5,0.7,0.9)$, and type of situation (low, medium, and high criticality).

All main effects were significant. Providing the system confidence information affected the verification rates, since there was a main effect of cue-availability, $F(1,13)=5.18$, $p<.05$. Effects were also significant for confidence level, $F(2,26)=13.31, p<.001$, and situation type $F(2,26)=136.10$, $p<.001$. There was an interaction between cue-availability and confidence level, $F(2,26)=14.04, p<.01$. Finally, the interaction between cue-availability and the type of situation was also significant, $F(2,26)=4.57, p<.05$. No other interactions reached statistical significance.

\section{DISCUSSION}

Experiment 2 shows that there is a significant effect on the verification rates when system confidence information is displayed. The results clearly indicate that users adapt to the system if system confidence is displayed. Participants verified the setting made by the context-aware system less often when the confidence of the system was high or medium (confidence levels of 0.9 and 0.7 ). When system confidence was low this effect disappeared (confidence level of 0.5 ), indicating that people inherently assume a confidence of above 0.5 and thus felt the need to verify the system state more often. This shows that the people can deal better with contextaware systems when they know how reliable they are - at least for medium and high levels of system confidence.

Experiments with similar objectives have also been carried out in domains with very high costs, such as air traffic control and military pilot training $[1,9,19]$. Here the subjects are highly-trained individuals that have practiced dealing with uncertainty information. In our experiments we show that equivalent results can be achieved with untrained individuals.

\section{CONCLUSION AND OUTLOOK}

For context-aware systems we often cannot assume that systems are highly reliable since context information is often incomplete, inaccessible, and uncertain. When interacting with such systems the notion of trust is one of the main factors of interest. While there have been proposals made to deal with ambiguous and uncertain context information through various feedback and control mechanisms, the effects on user's trust are hardly known since they are rarely evaluated. In this paper, we propose a simple but effective feedback mechanism of displaying system confidence. The effectiveness of the feedback mechanism is shown.

In our experiments we use a set of videos from everyday situations to examine the use of a context-aware mobile phone. Our experiments show that when system confidence is displayed, users more often rely on the system. This suggests an increase of the user's trust in the system.

Obviously many issues remain to be addressed. For research on context-aware mobile phones a deeper analysis of the results of the first experiments remains open. The (dis-) agreement of users on appropriate notification modalities and criticality levels are highly interesting, because they offer insight into how much a system has to adapt to it's current user and how much it can be 'ready-made'.

The display of system confidence information is mainly of interest where systems are used in diverse and changing settings, e.g. mobile applications that rely on context information. For such scenarios the graphical display of system confidence is very well suited. Graphical displays (at least given today's devices) do not provide information that is available at a glance. Better suited would be displays that provide an ambient awareness of the systems currect confidence. Ideally such a display would be 'readable' without being (socially) disruptive for the user and his environment.

Most approaches for mobile awareness displays actively try to grab the user's attention. Wisneski [28] proposes to use different channels such as heat, vibration, and physical expansion to convey information to the user. Other approaches use fine-grained vibration patterns (e.g. ActiveTouch [23]) or spatialized sound [24] to provide an awareness of information. Used on a regular basis an awareness display should however be a passive display that does not change when the displayed information is not changing. The promise is that a passive display will take less of the user's attention, especially when it is unnecessary to do so. 


\section{REFERENCES}

[1] S. Banbury, S. Seldcon, M. Endsley, T. Gordon, and K. Tatlock. Being certain about uncertainty: How the representation of system reliability affects pilot decision making. In Proc. Human Factors and Ergonomics Society 42nd Annual Meeting, 1998.

[2] V. Bellotti and K. Edwards. Intelligibility and accountability: Human considerations in context-aware systems. Human-Computer Interaction, 16(2):193-212, 2001.

[3] C. Bubb-Lewis and M. Scerbo. Getting to know you: Human-computer communicationin adaptive automation. In M. Mouloua and J. Koonce, editors, Human-Automation Interaction: Research and Practice. Erlbaum Associates, 1997.

[4] M. Chalmers and I. MacColl. Seamful and seamless design in ubiquitous computing. In Workshop: At the Crossroads: The Interaction of HCI and Systems Issues in Ubicomp, 2003.

[5] P. de Vries, C. Midden, and D. Bouwhuis. The effects of errors on system trust, self-confidence, and the allocation of control in route planning. International Journal of Human-Computer Studies, 58(6):719-736, 2003.

[6] M. Dzindolet, S. Peterson, R. Plmranky, L. Pierce, and H. Beck. The role of trust in automation reliance. International Journal of Human-Computer Studies, 58(6):697-718, 2003.

[7] M. Eidelkind and S. Papantonopoulos. Operator trust and task delegation: Strategies in semi-autonomous agent systems. In M. Mouloua and J. Koonce, editors, Human-Automation Interaction: Research and Practice. Erlbaum Associates, 1997.

[8] J. Fogarty, S. E. Hudson, and J. Lai. Examining the robustness of sensor-based statistical models of human interruptability. In Proc. ACM CHI, pages 207-214, 2004.

[9] K. Gempler and C. Wickens. Display of predictor reliability on a cockpit display of traffic information. Technical Report ARL-98-6/Rockwell-98-1, Aviation Research Lab, Institute of Aviation, University of Illinois, 1998.

[10] S. Greenberg. Context as a dynamic construct. Human-Computer Interaction, 16(2):257-268, 2001.

[11] E. Horvitz. Principles of mixed-initiative user interfaces. In Proc. CHI, pages 159-166, 1999.

[12] E. Horvitz and M. Barry. Display of information for time-critical decision making. In Proc. 11th Conf. on Uncertainty in Artificial Intelligence, pages 296-305. Morgan Kaufmann, 1995.
[13] N. Kern, S. Antifakos, B. Schiele, and A. Schwaninger. A model of human interruptability: Experimental evaluation and automatic estimation from wearable sensors. In Proc. ISWC, pages 158-165, Washington DC, USA, Nov 2004.

[14] N. Kern and B. Schiele. Context-aware notfication for wearable computing. In Proc. ISWC, pages 223-230, White Plains, NY, USA, October 2003.

[15] P. Maes. Agents that reduce work and information overload. Communications of the ACM, 37:30-40, 1994.

[16] J. Mankoff, S. Hudson, and G. Abowd. Interaction techniques for ambiguity resolution in recognition-based interfaces. In Proc. UIST, 2000.

[17] B. Muir. Trust in automation: Part i. theoretical issues in the study of trust and human intervention in automated systems. Ergonomics, 37(11):1905-1922, 1994.

[18] A. Newberger and A. Dey. System support for context monitoring and control. In Workshop: At the Crossroads: The Interaction of HCI and Systems Issues in Ubicomp, 2003.

[19] D. Nicholls, V. Barsotti, P. Battino, P. Marti, and S. Pozzi. Presenting uncertainty to pilots and controllers. In 5th Intern. Seminar on ATM, FAA, and EuroControl, 2003.

[20] E. Oztop, D. Wolpert, and M. Kawato. Mental state inference using visual control parameters. Cognitive Brain Research, (22):129-151, 2005.

[21] R. Parasuraman. Human use and abuse of automation. In M. Mouloua and J. Koonce, editors, Human-Automation Interaction: Research and Practice. Erlbaum Associates, 1997.

[22] D. Patterson, L. Liao, D. Fox, and H. Kautz. Inferring high-level behaviour from low-level sensors. In Proc. UbiComp, 2003.

[23] I. Poupyrev, S. Maruyama, and J. Rekimoto. Ambient touch: Designing tactile interfaces for handheld devices. In Proc. UIST, 2002.

[24] N. Sawhney and C. Schmandt. Nomadic radio: speech and audio interaction for contextual messaging in nomadic environments. ACM Transactions on Computer-Human Interaction, 7(3):353-383, 2000.

[25] T. Sheridan. Computer control and human alienation. In Technology Review, pages 61-73, 1980.

[26] S. Tiernan, E. Cutrell, and M. Czerwinski. Effective notification systems depend on user trust. In Proc. Human-Computer Interaction - Interact, pages 684-685, 2001.

[27] J. Williamson and R. Murray-Smith. Sonification of probabilistic feedback through granular synthesis. IEEE Multimedia, 12(2):45-52, April/June 2005.

[28] C. Wisneski. The design of personal ambient displays. Master's thesis, MIT, 1999. Thesis Proposal M.Sc. 